\title{
Prostaglandin E
}

National Cancer Institute

\section{Source}

National Cancer Institute. Prostaglandin E. NCI Thesaurus. Code C157134.

A family comprised of three naturally occurring prostaglandins that are involved in the regulation of many biological functions including vasodilation, inflammation and smooth muscle cell contractility. 